\title{
USE OF THE MAGNETORESISTIVE MAGNETIC FIELD SENSORS FOR THE TESTING OF THE LINEAR INDUCTION MOTORS
}

\author{
Ashish Varshney \\ Vilnius Gediminas Technical University
}

\begin{abstract}
The magnetic field of an LIM is more complex than that of a rotating induction motor. At analysis of LIM the limited dimensions of the inductor should be considered. Therefore, it is necessary to investigate the magnetic fields beyond the inductor boundaries (edge effect and end effect), and interdependencies of these fields. Because of the phenomenon of end effect, additional factors must be considered: there is a reduction of attraction force, and despite a balanced supply voltage, increased phase impedance and phase differences of leading currents. The first task is to measure the magnetic field parameter of LIM by using a hall sensor and CMR.
\end{abstract}

\section{INTRODUCTION}

Single-phase drive has less efficiency, but can be utilized where there is a single-phase feeding network. Therefore, single-phase drive is more widely applied for domestic appliances; they can be used as controlled servo motors in automatic systems. Linear induction motors (LIM) are used in many different applications, from slow moving sliding doors to high-speed trains around the world. Anything that requires linear motion will require a LIM. The idea of a LIM had been suggested in 1895 and was first developed by an English Electrical Engineer, Eric Laith waite. He spent the rest of his career investigating these special machines. His colleagues are currently designing a launch system with linear motors, which is a more efficient alternative to the rockets used to launch spacecraft. The objective of the first task is to measure the magnetic field parameter of LIM by using hallsensor and CMR sensor.

The magnetic field of an LIM is more complex than that of a rotating induction motor. At analysis of LIM the limited dimensions of the inductor should be considered. Therefore, it is necessary to investigate the

\author{
Ayush Varshney \\ SRM Institute of Science and Technology
}

magnetic fields beyond the inductor boundaries, and interdependencies of these fields.

Three-phase linear drive is used more often in industry because it is characterized by greater power and efficiency; however, connection requires a three-phase feeding network. Single-phase drive has less efficiency, but can be utilized where there is a singlephase feeding network. Therefore, single-phase drive is more widely applied for domestic appliances; they can be used as controlled servo motors in automatic systems. The single-phase linear motor is sparsely discussed in literature sources. Its magnetic field distribution with respect to time outside of inductor limits has not been delineated. Present day technology can be used to facilitate the investigative process into this problem.

\section{AIM OF THE WORK}

The aim of this work involves investigation of possibilities to use the manganite-based magnetic field sensors for testing of linear induction motors by measuring the magnetic field characteristics.

\section{TASKS OF THE WORK}

1. To analyze the simulation and experimental investigation methods for characterization of magnetic fields of linear induction motors (LIM).

2. To investigate the magnetic fields generated by LIM by means of manganite-based magnetic field sensors without the secondary moving element (rotor) of LIM. 3. To investigate the magnetic field parameters inside the gap between the primary winding and Moving element of LIM.

4. To compare manganite-based sensor measurement results of magnetic fields with results obtained by the conventional Hall sensors.

5. Using the obtained results to evaluate the measurement errors and obtain the advantages and disadvantages of manganite-based sensors compared to Hall sensors. 


\section{International Journal of Engineering Applied Sciences and Technology, 2021 Vol. 6, Issue 3, ISSN No. 2455-2143, Pages 222-224 \\ Published Online July 2021 in IJEAST (http://www.ijeast.com)}

\section{THE OBJECTIVES}

1. To perform the analysis of magnetic fields generated by linear induction motors (LIM) and possibilities of using the manganite-based magnetoresistive magnetic field sensors for measuring of these fields.

2. To perform the experimental investigations of magnetic fields of LIM without secondary elements using manganite-based magnetic field sensors and conventional Hall sensors.

3. To investigate the magnetic fields of LIM with movable secondary elements using manganite-based magnetic field sensors and conventional Hall sensors. 4. To perform the obtained experimental results for evaluation of possibilities of manganite-based magnetic field sensors for testing of LIM with comparison to Hall sensors.

\section{RESULT}

The linear induction motor (LIM) was designed and produced in the Department of Automation of Vilnius Gediminas Technical University. The three-phase frequency converter was designed and produced in the Electronic System Laboratory of the Centre for Physical Sciences and Technology. The frequency converter is supplied by DC voltage. Digital controllers can regulate the output frequency with indicators from 1 to $50 \mathrm{~Hz}$ by $0.1 \mathrm{~Hz}$ step. The output $\mathrm{AC}$ voltage of the converter depends on DC voltage and frequency.

The values of $\mathrm{AC}$ and $\mathrm{DC}$ voltage were measured by means of multimeter TTi 1906 while the AC current was measured by contactless current meter UT200B. The frequency converter was loaded by LIM at threephase star connection and AC line voltage was measured. In this case phase voltage is 1.73 times lower. The results of frequency converter test is shown in Table 3.2

At $50 \mathrm{~Hz}$ measured phase current (RMS value) was approximately $6.6 \mathrm{~A}, 10.2 \mathrm{~A}, 15.0 \mathrm{~A}$ and $20.4 \mathrm{~A}$ at $100,150,200$ and $250 \mathrm{~V} \mathrm{DC}$ voltage of frequency converter supply.

\section{CONCLUSION}

- Linear electromagnetic motor was experimentally examined and dependence relations of the interlinked magnetic flux, inductance and traction force on the position of the secondary element, at different supply voltages of the motor, were obtained.

- Linear electromagnetic motor was experimentally examined and dependence relations of the interlinked magnetic flux, inductance and traction force on the position of the secondary element, at different supply voltages of the motor, were obtained.

- Magnetic flux density in the air gap varies more in cross-section near the inductor than in cross-section near the secondary element.

- We have developed a pulsed magnetic field measurement system based on the CMR-B-scalar sensors for investigations of electro-dynamic processes in electromagnetic launchers.

- The system can also be applied for the investigation of high-pulsed magnetic fields in the frequency range up to $20 \mathrm{kHz}$ generated by electromagnetic catapults or small explosive magnetic generators.

\section{REFERENCES}

[1]. M.S. Manna, S. Marwaha, C. Vasudeva, 2009, Air Gap Field Analysis of Single Sided Linear Induction Motor with Time Harmonic Finite Element Method, International Journal of Recent Trends in Engineering, Vol. 2 Issue 1, pp- 50-52.

[2] I. Boldea, S.A. Nasar ,1997, Linear Electric Actuators And Generators , Cambridge University Press, p.total 235.

[3]. Budig P. K.2000 The Application of Linear Motors // Proc. of 9-th Int. Conf. on Power Electronics and Motion Control. - Kosice, Slovak Republic. Vol. 11-P. 1336 - 1341.

[4]. Karaliunas B., Matkevicius E,2006 Calculation of the Parameters of Linear Electric Drive // Trans Tech Publications, Mechatronic Systems and Materials, MSM 2005.Switzerland,. - Vol. 113. - P. 34 - 38.

[5] Karaliunas B.,2008 Research on Electric and Magnetic Asymmetry of Linear and Arc Motors, ISSN 1392-1215 Electronics and electrical engineering. no.1(81) t 190 Vol. 114. - P. $39-41$.

[6]. www. arborsci.com/Data_Sheets/Files/Sensor_Books/Volta ge_Sensors.

[7].Zurauskiene,N.et al.,2011 B-Scalar Sensor Using CMR Effect in Thin polycrystalline Manganite Films, IEEE Transaction on Plasma Science, Vol. 39, No. 1: 411-416 (2011).

[8]. Zurauskienè, N. et al,2009 Influence of Sr Content on CMR Effect in Polycrystalline La1-xSrxMnO3 Thin Films, Acta Physica Polonica Vol 115: 11361138. 
[9]. Zurauskiene, N. et al,2014 Magnetoresistance and Resistance Relaxation of Nanostructured La-Ca-MnO Films in Pulsed Magnetic Fields, IEEE Transactions on Magnetics, Vol 50, Issue: 11.

[10]. Zurauskiene, N. et al,2010. Colossal Magnetoresistance Properties of La0.83Sr0.17MnO3 Thin Films Grown by MOCVD on Lucalox Substrate, J Low Temp Phys, 159: 64-67, Vol 50, Issue: 11.

[11]. T.Stankevic, M.Schneider, and S.Balevicius, 2013 Pulsed magnetic field measurement system based on colossal magnetoresistance-B-scalar sensors for rail gun investigation IEEE Trans. Plasma Sci. 41, 2790, Vol 50, Issue: 11.

[12]. S. Balevicius, V. Stankevic, N. Žurauskiene ,2013 B-scalar sensors for rail gun investigation, Acta Phys. Pol., A 115, 1133 (2009), http://przyrbwn.icm.edu.pl/APP/PDF/115/ a115z661.pdf.

[13].T.Stankevic, L.Medisauskas, V.Stankevic, S.Balevicius, N.Zurauskiene, O.Liebfried, M. Schneider, Pulsed magnetic field measurement system based on colossal magnetoresistance-B-scalar sensors for rail gun investigation, Rev. Sci. Instrum. 85 (2014) 044704.

[14].www.lakeshore.com/products/Gaussmeters hall probes/ Gaussmeter/ Pages/ Overview. aspx.

[15]. T. Sadauskas, 2007 et all Distribution of magnetic field., B. Karaliunas, Research of magnetic asymmetry, Electronics And Electrical Engineering. Nr. 4(76), vol no. 4(76). - P. 69-72.

[16]. T. Sadauskas, A. Smilgevicius, Z. Savickiene, 2007 Distribution of Magnetic Field of Linear Induction Motor // Electronics and Electrical Engineering. - Kaunas: Technology ,vol no. 4(76). P. 63-66.

[17]. B.Karaliunas, 2008 Research on Electric and Magnetic Asymmetry of Linear and Arc Motors // Electronics and Electrical Engineering. - Kaunas: Technology, - vol no. 1(81). - P. 61-64.

[18] M. Molis, E. Matkevičius, L. Radzevičius, 2008 The Experimental Research of the Linear Motor // Electronics and Electrical Engineering.- Kaunas: Technology,.vol.1 - No. 7(87) T190. - P. 57-60. 